\title{
Coronal hydromagnetic implosions
}

\author{
Å. M. Janse ${ }^{1,2}$ and B. C. Low $^{2}$ \\ ${ }^{1}$ Institute of Theoretical Astrophysics, University of Oslo, PO Box 1029, Blindern, 0315 Oslo, Norway \\ e-mail: a.m.janse@astro.uio.no \\ 2 National Center for Atmospheric Research, PO Box 3000, Boulder, CO 80307, USA
}

Received 23 March 2007 / Accepted 22 June 2007

\section{ABSTRACT}

\begin{abstract}
Aims. The released magnetic energy in coronal events, i.e. in flares and coronal mass ejections (CMEs), is believed to have been stored locally in the coronal magnetic field. The energy in a magnetic field $\boldsymbol{B}$ is distributed in space with density $\frac{B^{2}}{8 \pi}$ that is also the isotropic magnetic pressure at each point in space. A localized release of magnetic energy would therefore imply a localized reduction of magnetic pressure. Hence, such a release could lead to an implosion of the magnetic structure as its atmospheric surrounding pushes inward. Whether an implosion would take place immediately depends on how fast the released energy can escape, through optically-thin radiation, thermal conduction, hydromagnetic waves, and, the magnetic channeling of high-energy particles.

Methods. We determined whether an expansion or an implosion would occur when cylindrical tubes of twisted flux relaxed to lower energy states. Depending on the dynamical nature of the relaxation we assumed, relevant dynamical invariants were invoked to relate a particular end state to the given initial state.

Results. Comparing the initial and the end state, we found that when most of the liberated energy escaped the cylinder imploded. The results suggest that implosions may take place simultaneously with flares and CMEs.
\end{abstract}

Key words. magnetohydrodynamics (MHD) - Sun: magnetic fields - Sun: corona - Sun: flares

\section{Introduction}

Relatively long-lived hydromagnetic structures populate the million-degree hot solar corona. These structures sporadically undergo dissipation to heat the local plasma, often to extremely high temperatures within seconds or minutes such as during a flare (Aschwanden et al. 2001; Aschwanden 2004). The observed physical conditions in the corona suggest that the magnetic field is the principal in situ source of the energy released. The energy in a magnetic field $\boldsymbol{B}$ is distributed in space with density $\frac{B^{2}}{8 \pi}$ which is also the isotropic magnetic pressure at each point in space. Recently Hudson (2000) took the insightful note that a localized release of magnetic energy would imply a localized reduction of magnetic pressure. Thus, such a release could lead to an implosion of a magnetic structure as its atmospheric surrounding pushes inward. Let us refer to this implosion as the Hudson effect.

The release converts magnetic energy into thermal and kinetic energies as well as high-energy particles (Lin \& Hudson 1976; Dennis 1988; Aschwanden 2004; Zhang \& Chye Low 2005). Whether an implosion would occur immediately depends on how fast these three forms of energy can escape. These energies do escape readily, through optically-thin radiation, thermal conduction, hydromagnetic waves, and, the magnetic channeling of high-energy particles (Lin \& Hudson 1976). Thus, the Hudson effect is unavoidable as a general consequence of the open state of magnetic structures. In other words, following an energy release, sooner or later a relaxed magnetic structure is likely to become more compact spatially. This paper is a simple hydromagnetic study of the Hudson effect with a one-dimensional model.

We organize our paper as follows. In Sect. 2, we define the physical goals of our study of magnetic energy release. In Sect. 3, idealized calculations are presented to give insights into the manner the Hudson effect may occur in the relaxation of a cylindrical flux tube to lower energy states. To model the open expanse of the corona, we use cylindrical tubes of twisted magnetic field embedded in an unbound domain containing a uniform field parallel to the tubes. In Sect. 4, we give a discussion of the physical results in relation to coronal phenomena.

\section{Taylor relaxation in open hydromagnetic systems}

Sophisticated time-dependent hydromagnetic simulations are needed to treat magnetic-energy releasing processes in the corona. There is a diversity of such processes operating over a broad spectrum of spatial and temporal scales, including, in particular, the relaxation of thin magnetic flux tubes on very small scales that heats the corona as proposed by Parker (1994), the flares, and, the CMEs on the largest scales. The dynamics involved in each process is distinct and extremely complex, involving magnetic reconnection and topological changes, couplings among the different atmospheric layers, and, coupling between macroscopic and microscopic processes many of which not well understood at the present. Our goal in this study is not to deal with these complexities but to formulate simple qualitative implications of the Hudson effect that we may look for either in observations or in future hydromagnetic simulations as they become available. To keep our model physically simple in such a first study, we select the coronal thin flux tubes in the Parker theory for a mathematical analysis. To avoid directly dealing with magnetic reconnection and related dynamics, we adopt the approach of Taylor (1974) to get at useful implications from a comparison between plausible initial and relaxed, lower-energy endstates, subject to dynamical constraints imposed by the turbulent transition from one to the other. 
Consider a quiescent region in the low corona where vertically oriented magnetic fields have threaded through from the chromosphere below. Observations suggest that these fields have small-scale structures down to below spatial resolutions of about $10^{3} \mathrm{~km}$ as may be achieved with a good telescope. This field is probably composed, on those very small scales, of tubes of twisted flux stacked together, in more or less locally parallel orientations such as envisaged by Parker (1994). Let us idealize this field morphology to that of a uniform field in unbounded space exterior to an infinite number of separate cylindrical flux tubes of arbitrarily specified radii. Inside each tube is a one-dimensional, twisted field varying only with the distance from the axis of the cylinder. The locations of the cylinders in the uniform external field can be freely specified with the cylinders possibly touching one another along a line. As will be seen, it is easy to construct a cylindrical field in equilibrium within each cylinder such that the field continues smoothly into the external common uniform field.

Depending on the degree of twist in each of these cylindrical fields, it may or may not turbulently relax on its own as a result of hydromagnetic instability to liberate free magnetic energy. This liberation generally involves a turbulent reconnection between the twisted field across its cylindrical boundary and the external uniform field. As that interaction proceeds, the dynamical region grows in size to include the successive cylindrical flux tubes as they are encountered. An avalanche effect is possible (Lu \& Hamilton 1991).

Before we present our investigation of this model in the next section, we sort out some physical issues and anticipate some physical effects.

\subsection{Physical considerations}

Our idealized model assumes that the coronal medium is so tenuous that in the initial equilibrium state, the plasma is perfectly electrically conducting but has negligible pressure and weight. It is a local model in that the large scale topology of the coronal field and the boundary conditions at the far ends of each tube of magnetic flux are all outside our approximation.

In a real relaxation, the cylindrical symmetry of the the initial state is broken and a locally three-dimensional turbulent hydromagnetic state is produced in which magnetic tangential discontinuities would form. The high electrical conductivity of the corona does not allow significant dissipation of electric currents except at such discontinuities containing extremely high densities of currents (Parker 1994). In terms of magnetic evolution, this dissipation proceeds with a local reconnection of field lines to change the field topology. Magnetic helicity is a measure of magnetic twist. The total magnetic helicity in a volume $V$ is defined as

$H(V)=\int_{V} \boldsymbol{A} \cdot \boldsymbol{B} \mathrm{d} V$

where $\boldsymbol{A}$ is the vector potential of the magnetic field $\boldsymbol{B}$. This formula is dependent on the free gauge of $\boldsymbol{A}$, in general, requiring proper additional constructions to apply the concept of magnetic helicity (Moffatt 1978; Berger \& Field 1984; Low 2006). We refer the reader to these works and references therein for the technical details of these constructions. Because the dissipation of electrical currents is intermitten in space and in time, magnetic helicity is not destroyed to a first approximation but is transferred from one local flux system to another via field reconnection (Taylor 1974, 1986; Berger 1984). This violent process produces local heating of the plasma to high temperature, nonfluid acceleration of charged particles to high energy, and acceleration of the plasma to high fluid velocity. The physics of this highly turbulent state is formidable, but the following assumptions seem physically reasonable for our purpose.

Let a cylinder in our idealized model undergo this turbulent energy release "uniformly" along the entire cylinder, over scales large compared to the reconnection process. Then we expect the reconnection to initiate within the cylinder "everywhere" along the length of the cylinder and spread outward in the two independent directions perpendicular to the axis. This simplifying assumption is made to allow us to use a one-dimensional treatment. The energy release generates two fronts propagating out of the cylinder into its exterior as indicated in Fig. 1, a sketch of the field morphology before and after a flux tube relaxes. The circles represent cross sections of the cylindrical flux tubes surrounded by the background uniform field pointing parallel to the cylinders. For some of the flux tubes the sense of field-line twist is indicated with an arrow. The leading front $F_{2}$, traveling initially at the fast hydromagnetic wave speed, is a moving boundary separating the undisturbed equilibrium state ahead of the ideal, i.e., non-dissipative hydromagnetic disturbances originating from the relaxation. This front may rapidly evolve into a hydromagnetic shock. In the open atmosphere, hydromagnetic waves may carry away a significant part of the energy released. A front $F_{1}$ separates the ideal hydromagnetic region ahead of it from the region behind where field reconnections have changed the field topology. Dissipative heating characterizes this inner region. Both fronts eventually impact and initiate various hydromagnetic processes as they encounter successive cylindrical tubes of twisted fields.

Consider a single cylinder of twisted field of radius $R_{0}$ in equilibrium in an unbounded, uniform-field, exterior. Artificially impose an electrically perfectly conducting, rigid wall at $R=R_{1}$ within which the field is assumed to turbulently relax to a lowerenergy equilibrium state. We may construct this relaxed state from the initial state if we are given the dynamical invariants of the relaxation to connect the two. Such a calculation avoids a direct treatment of the relevant relaxation dynamics but offers the following physical insight. If $R_{1} \leq R_{0}$, either a partial inner part or the whole of the original flux tube would undergo relaxation. If $R_{1}>R_{0}$, the relaxed field would include a reconnection between the original flux tube and a boundary layer of the background uniform field in $R_{1}>R>R_{0}$. Keep in mind that the plasma is taken to be perfectly conducting except for the formation of current sheets during the relaxation. Ampere's law then ensures that the net axial field in $R<R_{1}$ is a conserved quantity. For the highly conducting plasma, the idea of Taylor (1974, 1986) provides another invariant based on the hypothesis that reconnection would liberate energy significantly faster than magnetic helicity can be destroyed. The end state must therefore conserve the total magnetic helicity residing in the initial state.

The original Taylor theory was developed for a field in a rigid, electrically perfectly conducting container. For our purpose we need to consider a direct interaction between the relaxing field with its surrounding magnetized plasma (Dixon et al. 1989). This is allowed for in our model by taking $R_{1}>R_{0}$. The moving front $F_{1}$ in Fig. 1, separating the turbulently resistive inner region from the ideal hydromagnetic disturbances propagating ahead of it, may be identified with the wall $R=R_{1}>R_{0}$. In an actual short-lived relaxation, $F_{1}$ at first rapidly sweeps up successive layers of uniform field but slows down in its outward motion when magnetic reconnection subsides. In such an actual development, the hydromagnetic front $F_{2}$ would have traveled 

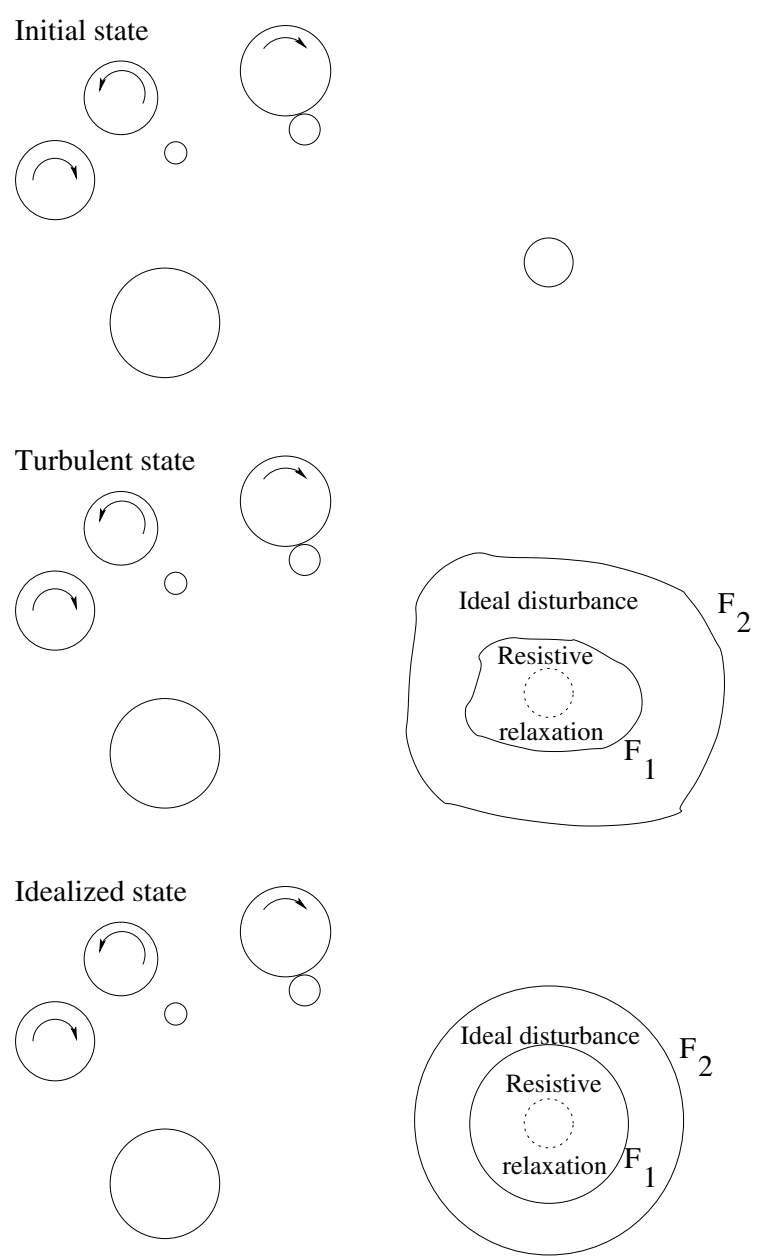

Fig. 1. Magnetic field configurations before and after a flux tube relaxes. An idealized state with cylindrical fronts is also included. The circles represent the cross sections of twisted, parallel cylindrical flux tubes, surrounded by uniform background field parallel to the cylinders.

into the far region. It leaves behind a near region where much of the turbulent reconnection is done while $F_{1}$ approaches a boundary in a fixed geometry and location. In our static analysis, the Hudson effect may be investigated by examining the possible end states defined by the appropriate dynamical invariants for a fixed $R=R_{1}$. If the total pressure of the end state at $R=R_{1}$ exceeds the uniform magnetic pressure of the exterior, a further outward expansion is indicated, i.e., this boundary if not rigid would move out. Conversely, if the the end state has less total pressure at $R=R_{1}$, an implosion is indicated.

In either case, taking $R=R_{1}$ to be a free fluid boundary rather than the assumed rigid wall will result in that boundary moving outward or inward, respectively, until equilibrium is established everywhere. Such an equilibrium may be achieved through ideal hydromagnetic displacements. We therefore consider next the ideal hydromagnetic equations for a onedimensional system.

\subsection{The ideal one-dimensional hydromagnetic equations}

Consider a hydromagnetic fluid in the unbounded domain to be described in cylindrical polar coordinates, varying only with $R$. The magnetic field is of the form

$\boldsymbol{B}=B_{\varphi} \hat{\varphi}+B_{z} \hat{z}$, describing helical lines of force winding on cylinders of constant $R$. For simplicity, we consider a polytropic fluid with index $\gamma$. Then, the ideal hydromagnetic equations governing this fluid are

$\rho\left(\frac{\partial v}{\partial t}+v \frac{\partial v}{\partial R}\right)=-\frac{\partial}{\partial R}\left(p+\frac{1}{8 \pi}\left[B_{\varphi}^{2}+B_{z}^{2}\right]\right)-\frac{B_{\varphi}^{2}}{4 \pi R}$,

$\frac{\partial \rho}{\partial t}+\frac{1}{R} \frac{\partial}{\partial R}(R v \rho)=0$,

$\frac{\partial B_{\varphi}}{\partial t}+\frac{\partial}{\partial R}\left(v B_{\varphi}\right)=0$

$\frac{\partial B_{z}}{\partial t}+\frac{1}{R} \frac{\partial}{\partial R}\left(R v B_{z}\right)=0$,

$\frac{\partial}{\partial t}\left(p \rho^{-\gamma}\right)+v \frac{\partial}{\partial R}\left(p \rho^{-\gamma}\right)=0$

where $p, \rho$ and $v$ are the plasma pressure, mass density and radial velocity, respectively.

In momentum Eq. (3), the two force densities on the right hand side are the force due to the sum of plasma and magnetic pressures and the magnetic tension force. The induction Eqs. (5) and (6) describe magnetic evolution in a perfect electrical conductor. The simplicity of this one-dimensional system allows us to represent the conservation laws for mass, entropy and magnetic flux explicitly in the Lagrangian description (Zel'dovich \& Raizer 1966). Suppose a Taylor relaxation has taken place in a twisted field inside a cylinder of radius $R_{1}$. If we take the subsequent evolution of the system to be ideal and one-dimensional, then that evolution is governed by Eqs. (3)-(7) with the conservation laws described below.

\subsection{Conservation laws}

Define the mass function

$M(R, t)=\int_{0}^{R} \rho R \mathrm{~d} R$,

giving the total mass in a unit-length cylinder of radius $R$. Then a direct integration of Eq. (4) gives

$\frac{\partial M}{\partial t}+v \frac{\partial M}{\partial R}=0$

to describe mass conservation. Similarly, we define the azimuthal magnetic flux for the unit-length cylinder as

$G(R, t)=\int_{0}^{R} B_{\varphi} \mathrm{d} R$

and the axial magnetic flux as

$F(R, t)=\int_{0}^{R} B_{z} 2 \pi R \mathrm{~d} R$

Then Eqs. (5) and (6) take the same form as Eq. (9), replacing $M$ with $G$ and $F$, respectively. Note further that Eq. (7) is of the same mathematical form as Eq. (9).

This derivation implies that $M, G, F$ and $S=p \rho^{-\gamma}$ are related to each other by specific single-variable functions. To see this, first note that $\rho>0$ implies $M(R, t)$ at any one time is a monotonically increasing function of $R$ and may thus be used as a, Lagrangian, coordinate in place of $R$. Write with no loss of generality $F(R, t)=F(M(R, t), t)$. Then, applying the advection 
Eq. (9) to $M$ and $F$ separately, it can be shown that $\frac{\partial F(M, t)}{\partial t}=0$. If we think of an initial value problem, giving $p, \rho$, and $\boldsymbol{B}$ at some initial time $t=0$, these initial values define the fixed functional forms $G(M), F(M)$, and $S(M)$ for all times. The distributions of $G, F$, and $S$ in space at subsequent times are given by the form of $M$ as a function of space and time according to Eq. (9) coupling $M(R, t)$ to $v(R, t)$. Hence, the mathematical problem has been reduced to one of determining these two dependent variables governed by Eq. (9) and the momentum Eq. (3). The latter takes the form

$$
\begin{aligned}
\frac{\partial v}{\partial t}+v \frac{\partial v}{\partial R}= & -\gamma\left(\frac{\mathrm{d} \sigma}{\mathrm{d} M}\right)^{\gamma-1}\left(\frac{1}{2 \pi R} \frac{\partial M}{\partial R}\right)^{\gamma-2} \frac{\partial}{\partial R}\left(\frac{\mathrm{d} \sigma}{\mathrm{d} M} \frac{1}{2 \pi R} \frac{\partial \sigma}{\partial R}\right) \\
& -\frac{1}{4 \pi} \frac{\mathrm{d} F_{z}}{\mathrm{~d} M} \frac{\partial}{\partial R}\left(\frac{1}{2 \pi R} \frac{\mathrm{d} F_{z}}{\mathrm{~d} M} \frac{\partial M}{\partial R}\right) \\
& -\frac{1}{2} \frac{\mathrm{d} F_{\varphi}}{\mathrm{d} M} \frac{\partial}{\partial R}\left(R \frac{\mathrm{d} F_{\varphi}}{\mathrm{d} M} \frac{\partial F_{\varphi}}{\partial R}\right)
\end{aligned}
$$

for fixed functional forms of $G(M), F(M)$, and $S(M)=$ $(\mathrm{d} \sigma / \mathrm{d} M)^{\gamma}$. This is a one dimensional hyperbolic system with information propagating strictly in the radial direction at the fast magnetosonic wave speed.

For application to a cylindrical flux tube surrounded by a uniform background field, the total energy per unit-length cylinder of radius $R$

$E=\int_{0}^{R} \frac{B^{2}}{8 \pi} 2 \pi R \mathrm{~d} R$,

is unbounded as $R \rightarrow \infty$. No expansion of the magnetized fluid to infinity is possible because it would involve an infinite amount of magnetic energy. This feature gives rise to an absolute confinement of a cylindrical flux tube within $R<R_{1}$ by the fields in $R \gg R_{0}$. We identify the flux tube with a spatially isolated solar coronal structure and the field in $R \gg R_{0}$ with the atmospheric surrounding of that structure. Expulsion of magnetized plasmas out of the atmosphere, such as seen in a coronal mass ejections, is entirely suppressed. While the internal mass before and after the relaxation may differ, as plasma enters and leaves the coronal portion of the flux tube, after the relaxation mass is conserved and Eq. (12) is valid.

There are two notable complications in the relaxation of a single cylindrical flux tube surrounded by a uniform field. First the wave front $F_{2}$ would propagate to infinity. If this front is a hydromagnetic shock, it would change the invariants $G(M)$, $F(M)$ and $S(M)$ of the local fluid as it sweeps across it. In the long-time limit, the waves associated with $F_{2}$ take away a part of the liberated excess energy. The remaining part is lost from the system by other mechanisms. In the long-time limit, then, a final set of invariants $G(M), F(M)$ and $S(M)$ determines the static equilibrium of the system. In the real corona, heating can readily drive siphon flows along a magnetic flux tube to change its total mass content, an important effect completely suppressed in our infinite-cylinder idealization. This could be ameliorated by allowing $M$, the mass between the cylindrical magnetic flux surfaces, to change in some parametrically prescribed way. For demonstrating the basic physics in our study, this sophistication does not seem well motivated. So we shall take the total mass in each cylindrical flux tube of our study to be conserved. But, the possibility of mass flows along flux tubes, such as commonly seen in post-flare magnetic loops, should be kept in mind in interpreting our results.

For the end state, $v=0$. Equation (9) is then trivially satisfied and Eq. (12) reduces to the static force balance equation to determine the mass function $M$ as a function of $R$ for invariants applicable to the evolution ending in the end state. Once $M(R)$ is known, the distributions of pressure and magnetic field are also known. The specification of our one-dimensional model is thus conceptually complete. Clearly, the final forms of the three invariants depend sensitively on the history of resistive reconnection and thermal processes, i.e., non-ideal processes, taking place in the evolution of the system. A number of theoretically conceivable possibilities can be considered without treating that evolution explicitly, as illustrated in the mathematical examples in the next section.

\section{Modeling local relaxation of a force-free field}

In the studies presented here we construct possible end states associated with an initial state of negligible pressure and gravitational force. The initial magnetic field (2) satisfies the equilibrium conditions

$$
\begin{aligned}
\nabla \times \boldsymbol{B} & =\alpha \boldsymbol{B}, \\
\nabla \cdot \boldsymbol{B} & =0,
\end{aligned}
$$

where $\alpha$ may be a function of space or a constant. These equations set the current density everywhere parallel to the field so the Lorentz force vanishes. In our one-dimensional system, the field has the form (2) and setting the Lorentz force to zero gives the equation

$\frac{\mathrm{d}}{\mathrm{d} R}\left(\frac{B_{\phi}^{2}+B_{z}^{2}}{8 \pi}\right)+\frac{B_{\phi}^{2}}{4 \pi R}=0$

showing that all cylindrical force-free fields are expressible in terms of $B^{2}=B_{\phi}^{2}+B_{z}^{2}$ treated as a generating function (Parker 1979). This equation shows that force-balance is achieved by a cancellation between the force due to the magnetic pressure $\frac{B^{2}}{8 \pi}$ and the magnetic tension force.

Taking examples of these initially force-free fields, we will consider for each the theoretically possible end states to examine the Hudson effect. We work with examples of increasing topological complexity, from single isolated flux tubes to the merging of two cylindrical flux tubes embedded in a uniform background field.

\subsection{Complete destruction of the field}

The first set of examples involves an initial state containing the force-free field of Gold \& Hoyle (1960)

$\boldsymbol{B}=B_{0} a\left(\frac{R}{a^{2}+R^{2}} \hat{\boldsymbol{\varphi}}+\frac{a}{a^{2}+R^{2}} \hat{z}\right)$,

where $B_{0}$ is the constant field amplitude and $a$ a constant length. This field is taken to fill all space. We will return to the case of a uniform background field after we have made a few basic points with this instructive case. Integration of the lines of force

$\frac{R \mathrm{~d} \varphi}{B_{\varphi}}=\frac{\mathrm{d} z}{B_{z}}$

results in

$z=a\left(\varphi-\varphi_{0}\right)$

where $\varphi_{0}$ is an integration constant. Each field line in the GoldHoyle field winds exactly the same number of turns per unit 


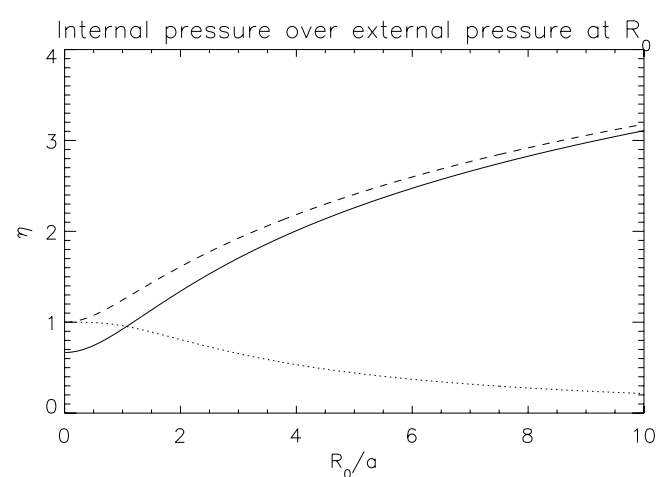

Fig. 2. The ratio $\eta$ as a function of $R_{0} / a$. The solid line represents the case of the complete destruction of the Gold-Hoyle field in $R<R_{0}$. The dotted line and the dashed line represent the situations where the Gold-Hoyle field in $R<R_{0}$ relaxes to a potential field and the released magnetic energy is completely lost via some suitable mechanism (dotted) or when it is converted into uniform thermal pressure (dashed).

length, independent of $R$. The quantity $1 / a$ is a measure of turns per unit length in the $z$-direction.

For illustrative purposes let us assume that the field is completely destroyed within a flux tube (infinite cylinder) of radius $R_{0}$, while the rest of the field, in $R>R_{0}$, remains unchanged. Suppose all the magnetic energy (per unit-length) of the tube

$E_{B}=\int_{0}^{R_{0}} \frac{B^{2}}{8 \pi} 2 \pi R \mathrm{~d} R$

is converted into thermal energy producing a uniform pressure $p_{0}$ within the cylinder,

$E_{\text {th }}=\int_{0}^{R_{0}} \frac{p_{0}}{\gamma-1} 2 \pi R \mathrm{~d} R=\frac{3 \pi}{2} R_{0}^{2} p_{0}$,

where $\gamma=5 / 3$ for a hydrogen plasma.

To determine whether the cylinder will explode or implode, we compare the uniform thermal pressure with the external magnetic pressure $p_{\mathrm{m}}$ at the cylinder surface, $R_{0}$. The pressure ratio $p_{0} / p_{\mathrm{m}}$ denoted by $\eta$, where

$\eta=\frac{2}{3} \frac{1+\left(R_{0} / a\right)^{2}}{\left(R_{0} / a\right)^{2}} \log \left(1+\left(R_{0} / a\right)^{2}\right)$,

is plotted as a function of $R_{0} / a$; see Fig. 2 . When $R_{0} / a<1.2$ the magnetic pressure $p_{\mathrm{m}}=\frac{B_{0}^{2}}{8 \pi} \frac{1}{1+\left(R_{0} / a\right)^{2}}$ is high, and the magnetic energy stored in the cylinder is not large enough to produce a uniform thermal pressure $p_{0}=\frac{B_{0}^{2}}{12 \pi} \frac{\log \left(1+\left(R_{0} / a\right)^{2}\right)}{\left(R_{0} / a\right)^{2}}$ as large as $p_{\mathrm{m}}$. As the field is destroyed in this hypothetical process, the cylinder will implode. For large ratios of $R_{0} / a$ the external magnetic pressure at the surface decreases, while the magnetic energy inside the cylinder increases to produce a thermal pressure larger than the external magnetic pressure. Then there would be an explosion.

\subsection{Case of a potential end state}

Next we assume that the field in $R<R_{0}$ relaxes into a potential state with the same total axial flux $F_{0}$ as the original GoldHoyle field. Then the axial magnetic flux is $F_{0}=\int_{0}^{R_{0}} B_{z} 2 \pi R \mathrm{~d} R=$ $\pi a^{2} B_{0} \log \left(1+\left(R_{0} / a\right)^{2}\right)$. The field after the relaxation is the uniform field

$\boldsymbol{B}_{\text {end }}=\frac{F_{0}}{\pi R_{0}^{2}} \hat{z}$ the potential state. Again, the external field in $R>R_{0}$ remains unchanged.

In the end state, the total pressure in the cylinder is $p_{0}+$ $\frac{B_{\text {end }}^{2}}{8 \pi}$, where $p_{0}$ is the uniform thermal pressure converted from the released magnetic energy, $\Delta E_{B}=E_{B \text {,initial }}-E_{B \text {, end }}$. The ratio between the internal and external pressures at $R=R_{0}$, following the relaxation, becomes

$$
\begin{aligned}
\eta & =\frac{1}{p_{\mathrm{m}}}\left(p_{0}+\frac{B_{\text {end }}^{2}}{8 \pi}\right) \\
& =\frac{2}{3} \frac{1+\left(R_{0} / a\right)^{2}}{\left(R_{0} / a\right)^{2}} \log \left(1+\left(R_{0} / a\right)^{2}\right)\left[1+\frac{1}{2} \frac{\log \left(1+\left(R_{0} / a\right)^{2}\right)}{\left(R_{0} / a\right)^{2}}\right]
\end{aligned}
$$

In Fig. 2 the ratio $\eta$ is shown to always be equal to or larger than 1 , thus the cylinder does not implode. When $R_{0} / a \rightarrow \infty$, the field amplitude in the end state, which is proportional to $\frac{\log \left(1+\left(R_{0} / a\right)^{2}\right)}{\left(R_{0} / a\right)^{2}}$, asymptotically approaches zero. In this limit, all the magnetic energy in the initial state is converted to thermal pressure, as in the previous section.

Up till now we have assumed that all released magnetic energy is converted into thermal pressure. What if the released energy instead is lost via some suitable mechanism? There would be no thermal pressure in the end state. Only a reduced magnetic pressure is found in $R<R_{0}$ and an implosion seems inevitable. In that case, the pressure ratio at $R=R_{0}$ becomes

$\eta=\frac{1+\left(R_{0} / a\right)^{2}}{\left(R_{0} / a\right)^{4}}\left[\log \left(1+\left(R_{0} / a\right)^{2}\right)\right]^{2}$.

The ratio $\eta$ is plotted as a function of $R_{0} / a$; see Fig. 2. For $R_{0} / a \sim 0$ the energy liberated, $\Delta E_{B}=$ $\frac{B_{0}^{2} a^{2}}{8} \log \left(1+\left(R_{0} / a\right)^{2}\right)\left[1-\frac{\log \left(1+\left(R_{0} / a\right)^{2}\right)}{\left(R_{0} / a\right)^{2}}\right], \quad$ is insignificantly small, and it does not make any difference whether the energy is turned into thermal pressure or lost. But for larger values of $R_{0} / a, \Delta E_{B}$ grows ( $a$ is constant), and the cylinder collapses. This is the basic point of the Hudson effect. Depending on what happens to the released magnetic energy, the flux tube will expand (the released energy is turned into thermal pressure) or collapse (the released energy escapes).

\subsection{Taylor relaxation}

Since magnetic helicity is conserved during reconnection under high electric conductivity, we next consider the physically more realistic relaxation of Taylor. Taylor relaxation has historically been applied to a closed magnetic field in a fixed finite domain with a vanishing normal field component at the boundary to assure a gauge-independent helicity (Taylor 1974, 1986). In our study Taylor relaxation must be applied to an infinite flux tube with care because the tubes total helicity $H(V)(1)$ is not gaugeinvariant, since the normal field component does not vanish at the "ends" of the tube. The relative helicity, introduced by Berger \& Field (1984), must be used instead of the total helicity. For our cylindrical flux rope, the total relative helicity per unit length of the rope is, in the notations of Hu et al. (1997) and Zhang et al. (2006),

$H_{R}=4 \pi \int_{R<R^{\prime}} \frac{A Q}{R} \mathrm{~d} R$ 
where the flux functions $A$ and $Q$ are related to the magnetic field in the following manner

$\boldsymbol{B}=\frac{1}{R}\left(Q \hat{\varphi}+\frac{\mathrm{d} A}{\mathrm{~d} R} \hat{z}\right)$

Equation (11) identifies $A$ to be the axial flux function $F$.

The end state following a Taylor relaxation is a force-free field with a constant $\alpha$; see Eq. (14). This is a field of minimum total magnetic energy for a given relative helicity (Berger 1985). In cylinder coordinates this minimum-energy state is the Lundquist field

$B_{z}=B_{\mathrm{e}} J_{0}\left(\alpha_{\mathrm{e}} R\right)$

$B_{\phi}=B_{\mathrm{e}} J_{1}\left(\alpha_{\mathrm{e}} R\right)$

where $R$ is the distance from the cylinder axis, $B_{\mathrm{e}}$ is the constant field amplitude, $J_{0}$ and $J_{1}$ are Bessel functions and $\alpha_{\mathrm{e}}$ denotes the constant value of $\alpha$. The two parameters $\alpha_{\mathrm{e}}$ and $B_{\mathrm{e}}$ are determined from conservation of the total axial flux $F_{0}$ and the relative helicity $H_{\mathrm{R}}$.

\subsection{Flux tubes embedded in a uniform field}

We return to the model with a uniform background field to treat the Taylor relaxation of an isolated flux tube of radius $R_{0}$. Suppose the field in the tube initially is a modified Lundquist field defined as

$$
\begin{aligned}
& B_{z}=\sqrt{B_{0}^{2} J_{0}^{2}\left(\alpha_{0} R\right)+B_{1}^{2}} \\
& B_{\phi}=B_{0} J_{1}\left(\alpha_{0} R\right)
\end{aligned}
$$

where again $R$ is the radial distance from the cylinder axis, $B_{0}, B_{1}$ and $\alpha_{0}$ are constants and $J_{0}$ and $J_{1}$ are the Bessel functions. The modified Lundquist field is a solution of the force-free Eqs. (14) (15) for a spatially varying $\alpha=\alpha_{0} \frac{B_{0} J_{0}\left(\alpha_{0} R\right)}{B_{z}(R)}$ for some suitable constant $\alpha_{0}$.

This initial field extends continuously into the external field

$$
\boldsymbol{B}=B_{2} \hat{z},
$$

in $R>R_{0}$ where $B_{2}=B_{z}\left(\alpha_{0} R_{0}\right)$ such that $\alpha_{0} R_{0}=3.8$, the first zero of the $J_{1}$ Bessel function. In this initial state, the thermal pressure is negligible and the magnetic field is continuous, so the cylinder surface is initially in pressure balance.

Following a Taylor relaxation, let the relaxed field occupy region $R<R_{1}$ where $R_{0}<R_{1}$. The front $F_{1}$ in Fig. 1 may be said to have included a part of the uniform field initially outside the original flux rope $R<R_{0}$. This relaxed field is a constant- $\alpha$ Lundquist field such that the total axial flux and the total relative helicity in $R<R_{1}$ are both conserved. These two conserved quantities determine the two free constant parameters $B_{\mathrm{e}}$ and $\alpha_{\mathrm{e}}$ defining the minimum-energy Lunquist field in $R<R_{1}$ as in the classical Taylor theory. This field is not continuous with the uniform field in $R>R_{1}$, in general. If the boundary $R=R_{1}$ (we have prescribed in an ad hoc manner) is removed, two dynamical consequences are produced. The discontinuity in the field at $R=R_{1}$ represents a current sheet on that cylinder that must dissipate resistively despite the large and finite electrical conductivity. Secondly, that boundary will move radially inward or outward as the result of the imbalance between the total pressures on the two sides of $R=R_{1}$. This suggests that in the open system, once relaxation begins in a flux tube, the reconnected field may continue to interact with its global surrounding. But the stored energy is locally of finite amount so that even though magnetic reconnection does not end abruptly as we have simplified in our static analysis, the reconnection activity must fall to a negligible level by the time the bulk of the energy has been released and escape from the system.

What our static analysis captures is that the outward expansion or inward collapse in such a realistic dynamical situation depends on the manner energy might escape after it has been released from a magnetic field. The time scales of these processes are essential. If the energy escapes sufficiently rapidly, an implosion is inevitable. Then, in addition to a final hydromagnetic wave $F_{2}$ in Fig. 1, there may even be a converging hydromagnetic wave within an imploding flux tube that perhaps would also steepen into a shock that compresses the plasma to high temperatures before the shock rebounds and moves out to the far regions, trailing behind the first front, $F_{2}$.

\subsection{Taylor relaxation of the modified Lundquist field, $R_{1}=R_{0}$}

The modified Lundquist field inside the cylinder, $R<R_{0}$, relaxes to a pure Lundquist field and releases free magnetic energy. If the external field remains unchanged, the pressure ratio between the internal and external total pressures at $R_{1}=R_{0}$, following the relaxation, is

$\eta_{\mathrm{rad}}=\frac{p_{L}}{p_{\mathrm{ext}}}$

if the released energy is lost via some appropriate mechanism, or

$\eta_{p_{0}}=\frac{p_{\mathrm{L}}+8 \pi p_{0}}{p_{\mathrm{ext}}}$

if the released energy is transformed into a uniform thermal pressure $p_{0}$. Here $p_{\mathrm{L}}$ and $p_{\text {ext }}$ are the pressures related to the Lundquist field and the external field, respectively.

The pressure ratios $\eta_{\mathrm{rad}}$ and $\eta_{p_{0}}$ as functions of $B_{1} / B_{0}$, as well as the energy ratio $E_{B \text {,end }} / E_{B \text {,initial }}$ are shown in Fig. 3 . When the quantity $B_{1} / B_{0}$ is small, the cylinder can undergo both an explosion and an implosion, depending on what happens to the released energy. If $B_{1}=0$ (equivalent to $B_{z}=\left|B_{0} J_{0}\left(\alpha_{0} R\right)\right|$ ) more than $1 / 4$ of the original magnetic energy is liberated and $\eta_{\mathrm{rad}}=0.85$ and $\eta_{p_{0}}=1.2$. As $B_{1} / B_{0}$ increases, the initial and end state become more alike, and for the extreme case of $B_{1} \gg B_{0}$ the initial and end state approach $\boldsymbol{B}=B_{1} \hat{z}$ and $E_{B \text {,end }} / E_{B \text {,initial }} \approx 1$. Thus as $B_{1} / B_{0}$ increases both pressure ratios $\eta_{\mathrm{rad}}$ and $\eta_{p_{0}}$ converge to unity. Though the model is highly simplified, it suggests that a coronal flux tube undergoing a relaxation may implode as long as the released energy escapes.

\subsection{Relaxation involving the background field with $R_{1}>R_{0}$}

When the front $F_{1}$ is at a distance $R_{1}>R_{0}$, a part of the uniform background field participates in the Taylor relaxation. Because the background field is a potential field, it has no magnetic twist. The background field only contributes to the total axial flux $F_{0}$, while the relative helicity $H_{\mathrm{R}}$, solely determined by the twisted cylindrical field, is invariant.

For $B_{1}=0$, the ratios $\eta_{\text {rad }}$ and $\eta_{p_{0}}$ at the relaxation front $R_{1}$ are plotted as a function of $R_{1} / R_{0}$; see Fig. 4. For large values of $R_{1} / R_{0}$ both $n_{\text {rad }}$ and $n_{p_{0}}$ asymptotically approach unity, while for smaller values the ratios reach their extreme values; $\eta_{\text {rad }}\left(1.14 R_{0}\right)=0.82$ and $\eta_{p_{0}}\left(1.22 R_{0}\right)=1.24$. For the case where the energy released during the relaxation completely escapes, the ratio $n_{\text {rad }}$ first decreases as the relaxation front propagates into 

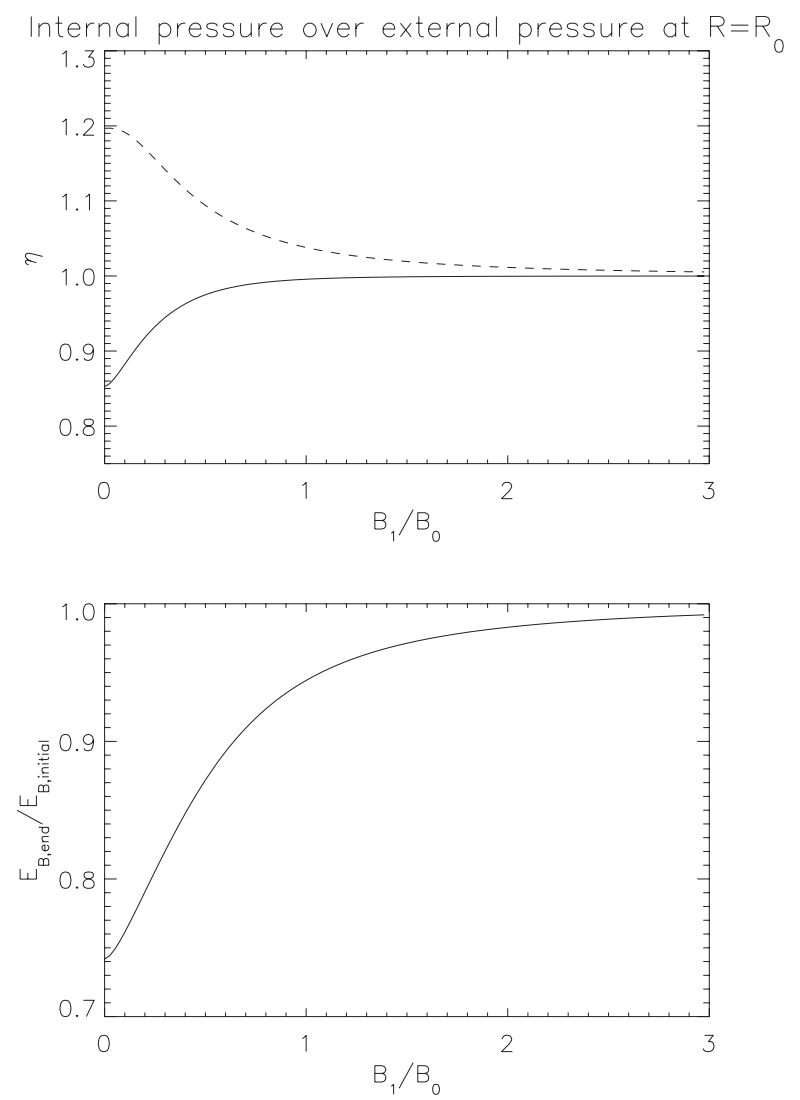

Fig. 3. The ratios $\eta_{\text {rad }}$ (solid line) and $\eta_{p_{0}}$ (dashed) and the end state energy over the initial state energy as a function of $B_{1} / B_{0}$, for the case of the flux tube undergoing a Taylor relaxation.

the external field. The total axial flux increases while the helicity remains unchanged. Hence, the helicity density decreases and more free magnetic energy is released. This results in an end state with lower energy density and lower magnetic pressure, and the ratio $\eta_{\text {rad }}$ decreases to its minimum value. But as more and more of the background field is included in the relaxation region, both the initial state and the end state closely resemble the external field, and $\eta_{\text {rad }}$ converges to one. Since $0.82 \leq \eta_{\text {rad }}<1$, there will be an implosion.

If the released energy instead is converted into uniform thermal pressure, the ratio between internal and external pressure at $R=R_{1}$ is $1<\eta_{p_{0}} \leq 1.24$, and the flux tube will expand. For small $R_{1} / R_{0}$ values the liberated energy increases faster than the area, producing higher and higher uniform thermal pressure $p_{0} \propto \Delta E_{B} / R_{1}^{2}$. The ratio $\eta_{p_{0}}=\eta_{\text {rad }}+8 \pi p_{0} / p_{\text {ext }}$ increases, despite the fact that $\eta_{\text {rad }}$ decreases. For larger values of $R_{1} / R_{0}$ the area increases faster than the liberated energy. Eventually the thermal pressure becomes insignificantly small and $n_{p_{0}} \approx n_{\text {rad }} \approx 1$.

Figure 4 also contains an energy ratio plot,

$\epsilon=\frac{E_{\mathrm{L}}-E_{\mathrm{pot}, \mathrm{L}}}{\left(E_{\mathrm{ML}}-E_{\mathrm{pot}, \mathrm{ML}}\right)}$

where $E_{\mathrm{L}}$ is the energy in the Lundquist field $R<R_{1}, E_{\mathrm{ML}}$ is energy in the modified Lundquist field $R<R_{0}$, and $E_{\text {pot,L }}$ and $E_{\text {pot,ML }}$ are the energy in a uniform, potential field with the same axial flux and radius as the field it is compared with, respectively the Lundquist and the modified Lundquist field. The ratio compares the excess energy above the potential energy for the end and the initial state. The energy in the background field is not included in the denominator since the background field, a
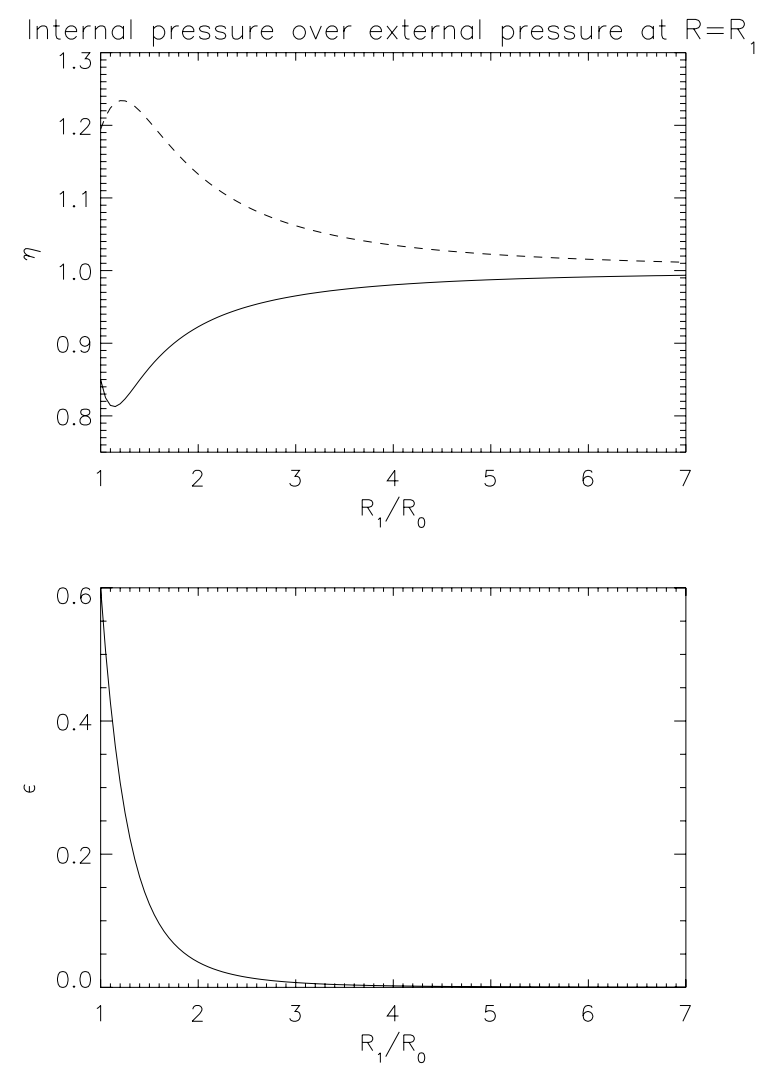

Fig. 4. The ratios $\eta_{\text {rad }}$ (solid line) and $\eta_{p_{0}}$ (dashed), in the upper panel, and $\epsilon$, in the lower panel, as a function of $R_{1} / R_{0}$, for the case of $B_{1} / B_{0}=$ 0 . Parts of the background field participates in the Taylor relaxation.

potential field, has no free magnetic energy. When $R_{1} / R_{0}$ increases, the end state approaches the potential state, and $\epsilon$ converges to zero.

As the front $F_{1}$ moves through the uniform background field (without crossing other flux tubes), the region behind this front will implode or explode depending on how much of the released energy is converted into thermal pressure. What happens when the front reaches another flux tube? A dynamical treatment is outside the scope of this paper, instead we investigate a simplified problem where two flux tubes are brought together and merge into one larger flux tube.

\subsection{Merging flux tubes and energy release}

Though the cases we study are one-dimensional, they can be used to simulate flux tube mergers in the corona since we are free to insert flux tubes at arbitrary locations in the uniform background. If two flux tubes were to merge and relax, how would this differ from the case where these two flux tubes went through isolated relaxations?

The two flux tubes are chosen to have the same radius $R_{0}$ and axial flux $F_{0}$, while they may have same helicity $H_{1}=$ $H_{2}$ or opposite helicity $H_{1}=-H_{2}$. The cylinders contain the modified Lundquist field (31)-(32) and are surrounded by the uniform field defined in (33). Suppose they merge, via some suitable process, and together with a part of the background field undergo a Taylor relaxation. The end state has a radius $2 R_{0}$, helicity $H_{1}+H_{2}$, and a flux $2 F_{0}+F_{\text {background }}$, where $F_{\text {background }}=2 \pi R_{0}^{2} B_{2}$. The energy released, when flux tubes of opposite and of same 
Table 1. Energy release in isolated and merging flux tubes with $B_{1}=0$ and $B_{1}=0.5 B_{0}$.

\begin{tabular}{|c|c|c|c|c|}
\hline Relaxation of & $\begin{array}{c}\epsilon_{1} \\
B_{1}=0\end{array}$ & $\begin{array}{c}\epsilon_{2} \\
B_{1}=0\end{array}$ & $\begin{array}{c}\epsilon_{1} \\
B_{1}=0.5 B_{0} \\
\end{array}$ & $\begin{array}{c}\epsilon_{2} \\
B_{1}=0.5 B_{0} \\
\end{array}$ \\
\hline Isolated tubes & 1.09 & 0.60 & 0.27 & 0.59 \\
\hline $\begin{array}{l}\text { Merging tubes of } \\
\text { same helicity }\end{array}$ & 0.06 & 0.08 & 0.02 & 0.08 \\
\hline $\begin{array}{l}\text { Merging tubes of } \\
\text { opposite helicity }\end{array}$ & 0 & 0 & 0 & 0 \\
\hline
\end{tabular}

helicity merge, is compared with the energy isolated tubes would release. Two ratios are calculated:

$\epsilon_{1}=\frac{E_{\text {end }}-E_{\text {pot,end }}}{E_{\text {pot,end }}}$

and

$\epsilon_{2}=\frac{E_{\text {end }}-E_{\text {pot,end }}}{\left(E_{\mathrm{ML}}-E_{\mathrm{pot}, \mathrm{ML}}\right)}$

where $E_{\text {pot,end }}$ and $E_{\text {pot,ML }}$ are the energy associated with a uniform field with the same axial flux and radius as the field it is compared with. $E_{\mathrm{ML}}$ is the initial energy in the two (isolated or merging) flux tubes, and $E_{\text {end }}$ is the end state energy. The first ratio indicates how close the end state is to the potential state, while the second ratio compares the excess energy above the potential energy for the end and the initial state.

The ratios $\epsilon_{1}$ and $\epsilon_{2}$ for isolated and merging tubes are shown in Table 1 . When $B_{1}=0$ the isolated flux tubes still have $60 \%$ of the excess energy left after the relaxation, whereas the merging tubes at most have $8 \%$ left. If the merging tubes have opposite helicity, the end state is the potential state, and all excess energy is released. While if the merging tubes have the same helicity, the end state has a nonzero helicity and cannot be a potential state. Still a very large part of the excess energy is liberated. Initially the helicity was contained within an area of $2 \pi R_{0}^{2}$, in the end state the helicity is spread out on an area twice as large, which lowers the helicity density and the energy of the end state. As $B_{1} / B_{0}$ increases the initial and the end state approach potential states, reducing the $\epsilon_{1}$ ratios, while the $\epsilon_{2}$ ratios are unaltered. The same amount of excess energy is released independent of the value of $B_{1}$, because both the numerator and the denominator in (38) are reduced.

How much energy do we expect each merger to release? If the released energy is of the same order as the potential energy and the fluxes tubes have a radius $R \sim 10^{3} \mathrm{~km}$ and a magnetic amplitude of $B \sim 2-20 \mathrm{G}$, in accordance with coronal values, then each merger releases

$\Delta E=2 \int_{V} \frac{B^{2}}{8 \pi} \mathrm{d} V=\frac{B^{2} R^{2} L}{4}=\frac{B^{2} R^{2} R_{\mathrm{s}}}{40}$,

where the flux tube length is assumed to be one tenth of the solar radius $R_{\mathrm{S}}, L=R_{\mathrm{S}} / 10$. For the chosen values each merger liberates $\sim 7 \times 10^{25}-7 \times 10^{27} \mathrm{ergs}$ (in the nanoflare/microflare range) or in energy per area, $\Delta E / 4 \pi R^{2}, 6 \times 10^{8}-6 \times 10^{10} \mathrm{erg} \mathrm{cm}^{-2}$.

\section{Discussion}

We conclude by examining the assumptions of Hudson in his conjecture that an implosion may take place simultaneously with a release of magnetic energy, and the implications these assumptions have for our model. The main results are summarized and we give a discussion of the limitations of the model, the physical results relating to coronal phenomena, and future studies.

It is widely accepted that the energy driving coronal transient events must come from the corona, because these events evolve rapidly at Alfvenic speeds that greatly exceed the speeds of photospheric motions. The slow photosphere may slowly build up coronal magnetic energy by stressing the fields in the corona. But, once a rapid energy release is triggered in the corona, the photosphere is too slow to contribute energetically to the release process. Furthermore the gravitational potential energy should not play a significant role. The dominance of the magnetic field in the corona in the above scenario is expressed by the smallness of its characteristic plasma $\beta$-factor, the ratio of the thermal pressure to the magnetic pressure.

Our static calculations indicated low values of $\beta$ that were never larger than 0.4 , when magnetic energy has been converted to a uniform plasma pressure. In the end state, the magnetic pressure is approximately constant in the entire region $R \leq R_{1}$, except in cases of low values of $B_{1} / B_{0}$ when we find a factor of 3 in the increase of magnetic pressure from $R=0$ to $R=R_{0}$. We should question our assumption that the released energy is converted all into a uniform thermal pressure. Since outside of reconnection regions the plasma is frozen into the magnetic field, the amount of plasma must vary greatly across the magnetic flux surfaces. We also remind ourselves that if siphon flows, suppressed in our one-dimensional models, are allowed, the plasma $\beta$ may be significantly different.

As a first study of the Hudson effect, we use Taylor relaxation to compare the initial and the end state of a twisted field inside a cylinder. When a significant part of the magnetic energy released during the relaxation is lost, e.g., through a combination of optically-thin radiation, thermal conduction, particle acceleration, and hydromagnetic waves, the thermal pressure in the end state is not large enough to compensate for the magnetic pressure reduction, which is related to the reduced magnetic energy density. The system would then, in principle, implode, as conjectured by Hudson. In addition to studying an isolated flux tube, we also treated the merging of two flux tubes into a larger flux tube, through a Taylor relaxation. The merger releases more magnetic energy than two isolated relaxations. Merging tubes of opposite twist relax to a potential state, and all the excess energy is liberated.

Among the several limitations of our model, we are lacking a detailed dynamical description of the interaction of the flux tube with the external field and, we are unable to determine what happens to the released energy without treating complex energetics and transport equations. Furthermore our model being not able to produce a continuous magnetic field at the boundary separating the relaxed region from the background field, strongly suggests that the instantaneous relaxation we have assumed is an oversimplification. In contrast to the problem with rigid walls, the relaxation never ends in the open system, except that the intensity of relaxation would wane as free energy becomes depleted.

Though the coronal magnetic field is highly complex and undergoes more violent dynamical events than the relaxation process we have studied, the models suggest that implosions can take place in the corona. Moreover, observations suggest that the released magnetic energy does readily escape. Studies of thermal and non-thermal energies of solar flares (Lin \& Hudson 1976; Saint-Hilaire \& Benz 2005) have found the non-thermal and the thermal energies to be of the same magnitude. During the flare impulsive phase, high energy, non-thermal electrons stream down the loop and deposit their energy in the lower corona and the chromosphere (footpoint-brightening, Krucker \& Lin 2002; 
Krucker et al. 2003). In addition energy will be lost through a combination of radiated, conduction, and mass motion. Thus, the flare region may implode as its atmospheric surrounding pushes inward.

Recently flare loop shrinking and loop height decrease have been observed (Forbes \& Acton 1996; Sui et al. 2004; Li \& Gan 2005, 2006). Using TRACE images, Li \& Gan (2006) found that the M2.5 flare on 2002 April 16 shrank about $30 \%$ early in the impulsive phase. The RHESSI 12-25 keV band and TRACE $195 \AA$ A images suggest a downward speed of respectively $13 \mathrm{~km} \mathrm{~s}^{-1}$ and $15 \mathrm{~km} \mathrm{~s}^{-1}$ for the loop-top. The contraction of the loop is followed by an expansion. Independent of the specific reconnection scenario, the Hudson effect requires the loop to initially shrink, as a vast amount of the liberated magnetic energy escapes. Later other process may dominated the evolution. Observations of inward moving waves do not necessarily support the idea of implosion. Inward moving waves, together with outward moving waves, may be produced by the reconnection event (Khan et al. 2006).

If indeed implosions are common, they may explain the high energy particles in terms of hydromagnetic shocks produced in flares, in CMEs and other magnetic energy release events. When a flux tube relaxes and consequently implodes, the inward moving hydromagnetic wave can steepened into a shock wave. The particles in the flux tube are then "trapped" in an imploding shock front sweeping particles into a collapsing volume. Particles having attained high enough energy will pass through the shock front, while particles with lower energies will repeatedly be reflected and accelerated by the front. The resulting particle energies will depend on how much of the energy in the shock front is transferred to the particles, as well as how the energy is distributed among the them. This interesting effect will be addressed in a future numerical study on the time-dependent hydromagnetic effects in the open system that lies outside the scope of our first study.

Acknowledgements. We thank Egil Leer for comments. The National Center for Atmospheric Research is sponsored by the National Science Foundation. This work was sponsored in part by the Research Council of Norway under grant 173792. BCL thanks Professor K. K. Phua, Director of the Institute of Advanced Studies and Professor S. Y. Lee for hospitality at the Nanyang Technological University.

\section{References}

Aschwanden, M. J. 2004, Physics of the Solar Corona. An Introduction (Physics of the Solar Corona)

Aschwanden, M. J., Poland, A. I., \& Rabin, D. M. 2001, ARA\&A, 39, 175

Berger, M. A. 1984, Geophys. Astrophys. Fluid Dynamics, 30, 79

Berger, M. A. 1985, ApJS, 59, 433

Berger, M. A., \& Field, G. B. 1984, Fluid Mechanics, 147, 133

Dennis, B. R. 1988, Sol. Phys., 118, 49

Dixon, A. M., Berger, M. A., Priest, E. R., \& Browning, P. K. 1989, A\&A, 225, 156

Forbes, T. G., \& Acton, L. W. 1996, ApJ, 459, 330

Gold, T., \& Hoyle, F. 1960, MNRAS, 120, 89

Hu, Y. Q., Xia, L. D., Li, X., Wang, J. X., \& Ai, G. X. 1997, Sol. Phys., 170, 283

Hudson, H. S. 2000, ApJ, 531, L75

Khan, J. I., Fletcher, L., \& Nitta, N. V. 2006, A\&A, 453, 335

Krucker, S., \& Lin, R. P. 2002, Sol. Phys., 210, 229

Krucker, S., Hurford, G. J., \& Lin, R. P. 2003, ApJ, 595, L103

Li, Y. P., \& Gan, W. Q. 2005, ApJ, 629, L137

Li, Y. P., \& Gan, W. Q. 2006, ApJ, 644, L97

Lin, R. P., \& Hudson, H. S. 1976, Sol. Phys., 50, 153

Low, B. C. 2006, ApJ, 646, 1288

Lu, E. T., \& Hamilton, R. J. 1991, ApJ, 380, L89

Moffatt, H. K. 1978, Magnetic field generation in electrically conducting fluids (Cambridge, England: Cambridge University Press), 353

Parker, E. N. 1994, Spontaneous current sheets in magnetic fields : with applications to stellar X-rays (New York : Oxford University Press)

Parker, E. N. 1979, Cosmical magnetic fields: Their origin and their activity (Oxford: Clarendon Press; New York: Oxford University Press), 858

Saint-Hilaire, P., \& Benz, A. O. 2005, A\&A, 435, 743

Sui, L., Holman, G. D., \& Dennis, B. R. 2004, ApJ, 612, 546

Taylor, J. B. 1974, Phys. Rev. Lett., 33, 1139

Taylor, J. B. 1986, Rev. Mod. Phys., 58, 741

Zel'dovich, Y. B., \& Raizer, Y. P. 1966, Physics of Shock Waves and High

Temperature Hydrodynamic Phenomena (New York: Academic Press)

Zhang, M., \& Chye Low, B. 2005, ARA\&A, 43, 103

Zhang, M., Flyer, N., \& Low, B. C. 2006, ApJ, 644, 575 